\title{
Proposal of information addition method to existing signs for autonomous driving
}

\author{
Koho Futatsuishi ${ }^{\mathrm{a}, 1}$, Lifeng Zhang a \\ ${ }^{a}$ Faculty of Engineering, Kyushu Institute of Technology \\ 1-1 Sensui-cho, Tobata-ku, Kitakyushu, Fukuoka, 804-8550, Japan \\ 1 futatsuishi.koho186@mail.kyutech.jp
}

\begin{abstract}
Automated driving technology is developing in recent years. High-level automatic driving uses radars, cameras, and other devices to grasp the surrounding environment's information. Recognition of road signs is one of them. However, in recognition of the traffic sign, there is a problem that a similar shape thing is erroneously recognized in the current method of judging by an external feature. This research proposes a system that recognizes road signs by reading a $\mathrm{QR}$ code made of a polarizing plate.
\end{abstract}

keywords: Image processing, Automatic driving, Polarizing film

\section{Introduction}

\subsection{Background}

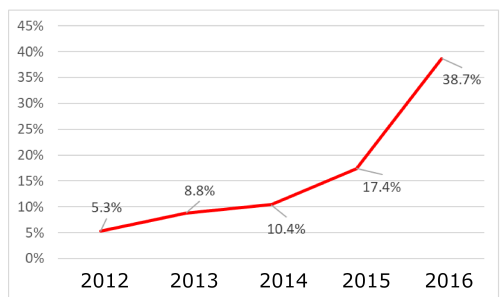

Fig. 1. Percentage of vehicles equipped with a function to follow the vehicle in front

In recent years, the spread and development of autonomous driving technology have been rapidly progressing. (1)

As shown in Fig:1, the percentage of vehicles equipped with distance control function to the previous vehicle has increased more than 7 times in Japan from 2012 to 2016.

In autonomous driving, which is a further advancement of these technologies, vehicles with the function of maintaining lanes, maintaining lanes, and changing lanes under driver monitoring conditions are being commercialized.
However, the combination of 3D maps, highly accurate sensors, and GPS information for a complete driving operation has been limited to testing and showing off concepts.

Such a high level of autonomous driving must eliminate false recognition by the system. Recognition of traffic signs is one of them. In the current method, the sign is recognized based on the external features from the camera image, so if there is something similar, there is a risk of misrecognition.

Therefore, it is considered that sign recognition that does not depend only on the shape is necessary for the nextgeneration autonomous driving technology.

\subsection{Purpose of research}

The purpose of this research is to develop a road sign recognition system using a polarizing film. Specifically, it is a system that detects the QR code that appears on the image of the camera by attaching a film on the existing sign and the in-vehicle camera and blocking the light. It is superior in terms of reliability compared to the conventional method without impairing the visibility of the sign from the driver. It is also considered superior in terms of cost compared to the proposal to standardize road signs for fully autonomous driving. (2)

Also, since it is possible to add information such as road surface and lane to the QR code, it is thought that it will improve the accuracy of automatic driving.

\section{Proposed method}

The processing flow of the proposed system is shown in Fig.2

First, each frame acquired from the camera image is smoothed, grayscaled, and binarized. The QR code position is detected from the image by the cascade classifier created by the machine learning algorithm called AdaBoost. 


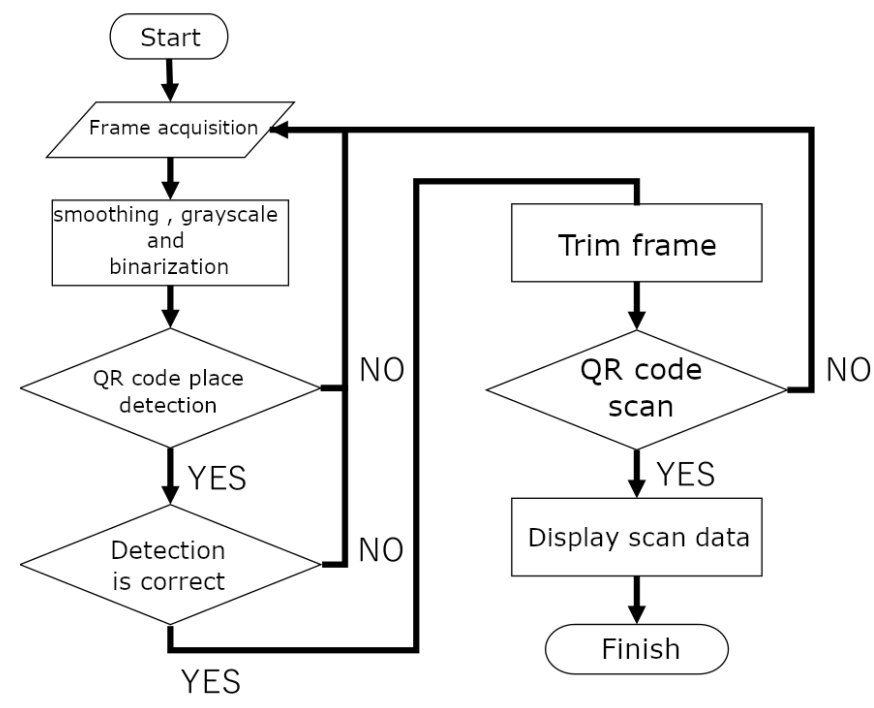

Fig. 2. Proposal system flowchart

The detected part is trimmed, and the data is $\operatorname{read}^{(3)}$ to recognize the road sign. Whether the $\mathrm{QR}$ code detection by the classifier is correct is judged by whether the difference between the coordinates of the recognized frame and the next recognized frame is within the threshold value. The polarizing film and the machine learning algorithm Adaboost are discussed in the next section.

\section{Principle}

\subsection{Polarizing plate}

Light is a transverse wave consisting of an electric field and a magnetic field that oscillates in directions perpendicular to each other in a plane perpendicular to the traveling direction. The surface containing the traveling direction of light and an electric or magnetic field is called an oscillating surface or a polarizing surface. The light is circularly polarized, and the vibrating surface of its components is rotating.

A polarizing plate is one in which the arrangement of molecules and the vibration direction of electrons are fixed in a fixed direction. Of the light incident on the polarizing plate, only the light whose vibration surface coincides with the electrons' vibration direction of the polarizing plate is transmitted. Therefore, as shown in Fig.3, when another polarizing plate is stacked to be orthogonal to one polarizing plate, the vibration plane of light passing through the first polarizing plate and the electrons of the second polarizing plate. The direction of travel is orthogonal, and light cannot pass through. ${ }^{(4)}$

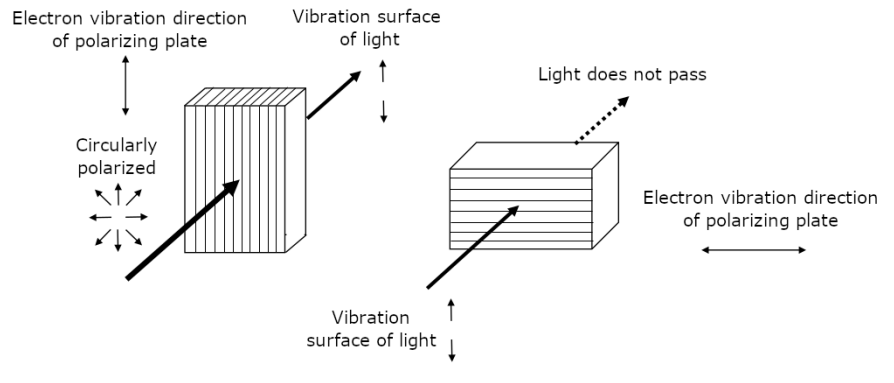

Fig. 3. Light passing through the polarizing plate

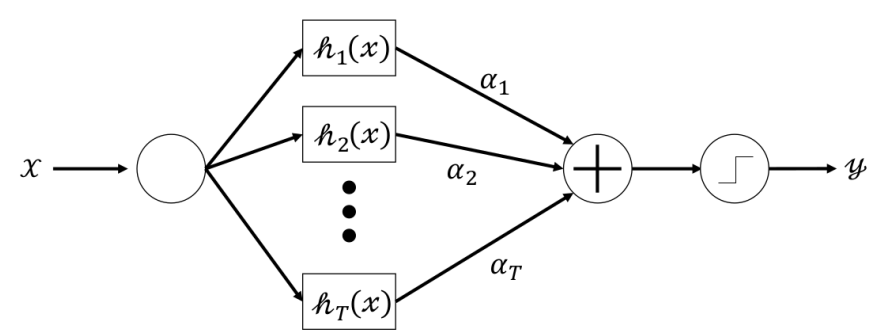

Fig. 4. The classifier obtained by adaboost

\subsection{AdaBoost}

Adaboost is one of supervised learning. When a training sample is given to Adaboost, a simple and different weak discriminator is created and trained while sequentially changing the weight of the training sample. A method of sequentially learning weak classifiers and increasing the accuracy of the classifiers is called Boosting.

Adaboost is a Boosting algorithm that adaptively updates sample weights and reuses samples. Fig. 4 shows the classifier obtained by Adaboost.

For the input pattern $x, T$ weak classifiers $h_{t}(x)$ are weighted with reliability $\alpha_{t}$ and combined to form a strong classifier, which outputs the label $y$ of the class to which the pattern belongs.

When learning, $h_{1}(x)$ is selected first, and then $h_{2}(x)$ is selected sequentially so that samples that are not good at $h_{1}(x)$ can be identified. ${ }^{(5)}$

\subsubsection{Algorithm}

As an example, as a weighted majority vote of $T$ weak classifiers $h_{t}(x)$, a strong classifier that finds the class label $y \in+1,-1$ of the unknown sample $x$ learns as follows.

Prepare $N$ training samples $\left(x_{1}, y_{1}\right), \ldots,\left(x_{N}, y_{N}\right)$, and a function $D_{t}(i)$ that evaluates the weight of each sample. $x_{i}$ is a sample, meaning the object you want to detect and other image patterns $y_{i}$ is a class label, labeled +1 for the object you want to detect, -1 otherwise. 
1. The weights of the initial sample are evenly distributed and initialized.

$$
D_{1}(i)=\frac{1}{N}
$$

2. At For $t=1, \ldots, T$

(A) Learn the weak classifier $h_{t}(x)$ in the sample distribution $D_{t}$.

Select ht that minimizes the error rate for the training sample. Error rate is calculated based on weight, not sample size

$$
\epsilon_{t}=\sum_{i: y_{i} \neq h_{i}\left(x_{i}\right)} D_{t}(i)
$$

(B) Calculate the reliability $\alpha_{t}$ from the error rate.

$$
\alpha_{t}=\frac{1}{2} \log \frac{1-\epsilon_{t}}{\epsilon_{t}}
$$

The smaller the error rate, the higher the reliability.

(C) Update the sample weights.

$$
D_{t+1}(i)=D_{t}(i) \exp \left[-\alpha_{t} y_{i} h_{t}\left(x_{i}\right)\right]
$$

Since $y_{i} h_{t}(x i)$ is 1 for the sample that was correctly recognized by the weak classifier, $D_{t+1}(i)=$ $D_{t}(i) \exp \left(-\alpha_{t}\right)$, and the weight becomes smaller.

For the wrong sample, since $y_{i} h_{t}(x i)=-1, D_{t+1}(i)=$ $D_{t}(i) \exp \left(\alpha_{t}\right)$, and the weight increases. When $\epsilon_{t}$ is 0.5 , $\alpha_{t}$ becomes 0 and the weights are not updated, so there is no need to continue learning.

(D)Normalize so that the sum of the sample weights is 1.

$$
\begin{gathered}
D_{t+1}(i)=\frac{D_{t+1}(i)}{Z_{t+1}} \\
Z_{t+1}=\sum_{i=1}^{N} D_{t+1}(i)=\sum_{i=1}^{N} D_{t}(i) \exp \left[-\alpha_{t} y_{i} h_{t}\left(x_{i}\right)\right]
\end{gathered}
$$

Weak classifiers with different fields of expertise are weighted by reliability and a majority vote is taken, and the final classifiers are as follows.

$$
H(x)=\sin \left[\sum_{t=1}^{N} \alpha_{t} h_{t}(x)\right]
$$

\subsubsection{Haar-like feature}

As shown in Fig.5, Haar-like feature expresses the feature value by the difference between the average brightness in the white square and the average brightness in the black square. All of these have features that enable high-speed calculation from the integrated image. Boosting a weak classifier based on Haar-like features to generate a classifier. This classifier produces a rejection type cascade.

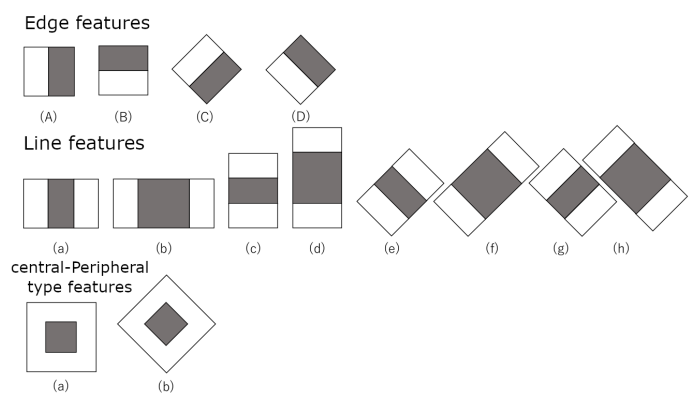

Fig. 5. Haar-like feature

\begin{tabular}{|c|c|}
\hline Effective pixels & 12 million pixels \\
\hline $\begin{array}{l}\text { Focal length } \\
\text { ( } 35 \mathrm{~mm} \text { equivalent) }\end{array}$ & $26 \mathrm{~mm}, 52 \mathrm{~mm}$ \\
\hline F-number & $\begin{array}{l}\text { F1.8 }(26 \mathrm{~mm}), \\
\text { F2.0(52mm) }\end{array}$ \\
\hline
\end{tabular}

Table 1. PC specifications

\begin{tabular}{|c|c|}
\hline Processor & $\begin{array}{l}\text { Intel(R)Core(TM) i5-3330 } \\
\text { CPU @ 3.00GHz 3.00GHz }\end{array}$ \\
\hline Memory & $8.00 \mathrm{~GB}$ \\
\hline
\end{tabular}

Table 2. Specifications of polarizing plate

\begin{tabular}{|c|c|}
\hline Thickness & $0.2 \mathrm{~mm}$ \\
\hline Transmittance & $40 \%$ \\
\hline Polarization rate & $99 \%$ or more \\
\hline
\end{tabular}

Table 3. Specifications of each experimental photography equipment
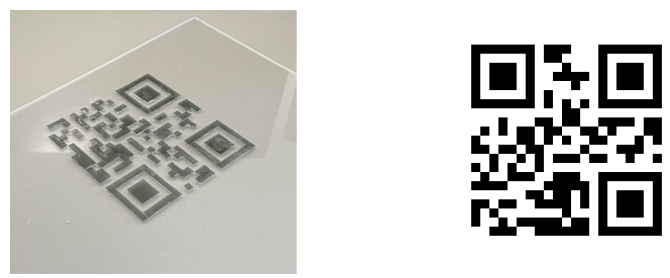

Fig. 6. Created QR code and Original QR

\section{Experiement}

\subsection{Experiment environment}

The specifications of the devices and instruments used in this experiment are as follows.

As shown in Fig.6, QR code was created on a transparent acrylic plate using a polarizing film. Its specifications were shown in Table 2.

\subsection{Cascade classifier}

As the correct image of the classifier, we use four types of images in which the QR code shown on the right in Fig.6 is rotated every 90 degrees. 
Table 4. Parameter setting of createsamples and number of generated sheets

\begin{tabular}{|c|c||c|c|}
\hline bgcolor & 255 & w & 24 \\
\hline maxxangle, maxyangle & 0.5 & h & 24 \\
\hline maxzangle & 0.3 & generated images & 5578 \\
\hline
\end{tabular}

Table 5. Parameter setting of "traincascade"

\begin{tabular}{|c|c||c|c|}
\hline numPos & 4500 & featureType & HAAR \\
\hline numNeg & 1250 & minHitRate & 0.995 \\
\hline numStage & 20 & maxFalseAlarmRate & 0.5 \\
\hline
\end{tabular}

The "createsamples" command is an OpenCV application, synthesizes correct images into incorrect images (1538 data) while rotating them at an angle that can be set by parameters.

The detailed parameter settings of "createsamples" and the number of images generated are shown in Table 4.

"maxxangle, maxyangle, maxzangle" mean to rotate (unit: radians) at random angles within this value for each $x, y, z$ axis. 'w' and 'h' represent the width and height (unit: pixels) of the generated sample image. ${ }^{(6)}$ A training vector file is generated based on the generated correct and incorrect images. Based on this, learn with the application "traincascade". The "traincascade" parameters are set as shown in Table 4.2

Set numPos and numNeg to 80 to 90 of the number of correct and incorrect images. "featureType" represents the type of feature used,'minHitRate' represents the target detection rate at each learning stage, and "maxFalseAlarmRate" represents the false positive rate.

\section{3 detectMultiScale}

Use the CascadeClassifier object detectMultiScale() method to detect the QR code in the image. Since it has multiple arguments to judge detection, the value was changed and the number of detections and the number of false positives were measured to obtain the optimum value for this study. ${ }^{(7)}$ As shown in Fig.7, the video used for evaluation was taken while walking from a point 10 meters away to a sign covered with a $\mathrm{QR}$ code made of polarizing film.

The two arguments that change the value here are scaleFactor and minNeighbors. The scaleFactor determines the amount of scale-up between each scale, and the larger the scale factor, the faster the calculation, but the detection may be overlooked. minNeighbors suppresses false detection by assuming that there is only one object at that position even if there are detections that overlap up to the set number of times.

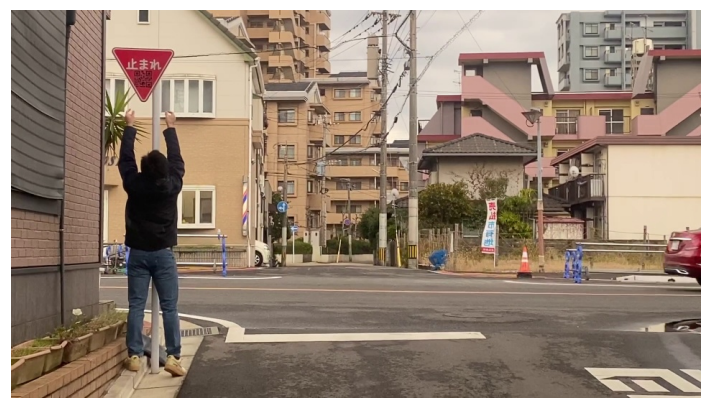

Fig. 7. Video for evaluation

This is a countermeasure against the tendency of the classifier to falsely detect an object even in the pixels and scale around the object to be detected. As a result of the verification, it was assumed that the optimum result could be obtained when scaleFactor $=1.74$ and minNeighbors $=7$. As a result of a part of the verification, when minNeighbors $=7$ and the scale Factor was changed from 1.71 to 1.79 , it was as shown in Table 6.

\subsection{Experiment 1}

An acrylic plate was placed over the traffic sign, and multiple videos were recorded while approaching from a point 10 meters away from the sign (focal length $52 \mathrm{~mm}$ ). Detection and reading were performed using these moving images. The outline of the video is shown in Table 7.

The location of the QR code could be detected in the daytime videos except the video ' $H$ ', but the video 'I' taken at night could not be detected. In the videos ' $E$ ' and ' $F$ ', data could be read in addition to position detection. Based on this result, the following experiment 2 was conducted.

\subsection{Experiment 2}

In Experiment 1, as a result of shooting and verifying videos in various environments, we were able to read data in some videos. In Experiment 2, we verified how many meters the data could be read at multiple resolutions. The picture was taken in an environment where the signboard is in a low position and is not exposed to direct sunlight shown in Fig.8. The video was shot moving 10 meters backwards from a location where 1.5 meters away from the sign.

\subsection{Consideration}

\subsubsection{Experiment 1}

Of the videos taken during the day, the position of the QR code could be detected using the cascade classifier for the videos other than video $H$. For video $H$, a polarized $Q R$ was 
Table 6. Measurement result at minNeighbors $=7$

\begin{tabular}{|c||c|c|c|c|c|c|c|c|c|}
\hline scaleFactor & 1.71 & 1.72 & 1.73 & 1.74 & 1.75 & 1.76 & 1.77 & 1.78 & 1.79 \\
\hline detected frames & 78 & 66 & 91 & 99 & 75 & 85 & 78 & 80 & 68 \\
\hline falsely detected framese & 27 & 46 & 26 & 5 & 25 & 16 & 23 & 16 & 18 \\
\hline
\end{tabular}

Table 7. Video overview

\begin{tabular}{|c|c|c|}
\hline & overview & Type of sign \\
\hline A & Daytime & no parking \\
\hline B & Daytime & $\begin{array}{l}\text { Prohibition of traveling } \\
\text { outside the designated direction }\end{array}$ \\
\hline $\mathrm{C}$ & Daytime & $\begin{array}{c}\text { Prohibition of traveling } \\
\text { outside the designated direction }\end{array}$ \\
\hline $\mathrm{D}$ & $\begin{array}{l}\text { Daytime } \\
\text { direct light }\end{array}$ & no parking \\
\hline $\mathrm{E}$ & Daytime & no parking \\
\hline $\mathrm{F}$ & Daytime & no parking \\
\hline G & Daytime & Speed Limit \\
\hline $\mathrm{H}$ & Daytime & School zone \\
\hline I & Night & no parking \\
\hline
\end{tabular}

Table 8. Results of Experiment 1

\begin{tabular}{|c|c|c|}
\hline & Total frames & Detected frame \\
\hline A & 295 & 44 \\
\hline B & 189 & 36 \\
\hline C & 360 & 97 \\
\hline D & 406 & 92 \\
\hline E & 329 & 28 \\
\hline F & 334 & 56 \\
\hline G & 446 & 19 \\
\hline H & 406 & 0 \\
\hline I & 316 & 0 \\
\hline
\end{tabular}

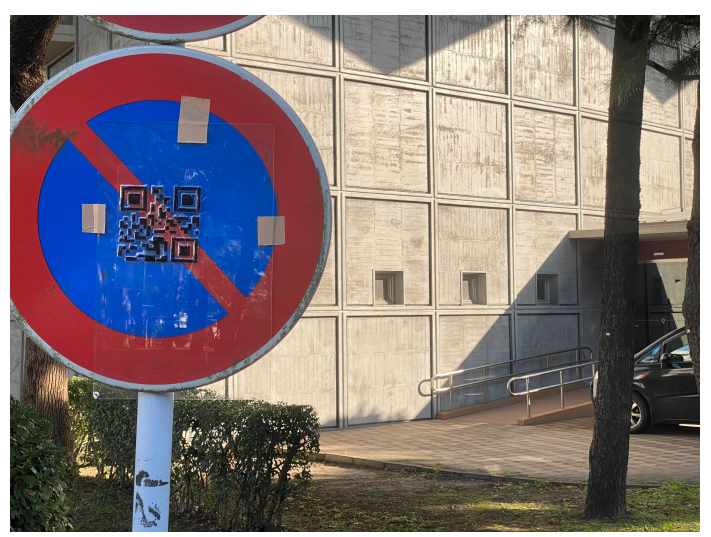

Fig. 8. Environment of Experiment 2

placed on the school road sign shown in Fig9. It is thought
Table 9. Results of Experiment 2

\begin{tabular}{|c|c|}
\hline Video resolution & Readable distance (maximum) \\
\hline $1920 \times 1080$ & $5.8 \mathrm{~m}$ \\
\hline $3840 \times 2160$ & $6.3 \mathrm{~m}$ \\
\hline
\end{tabular}

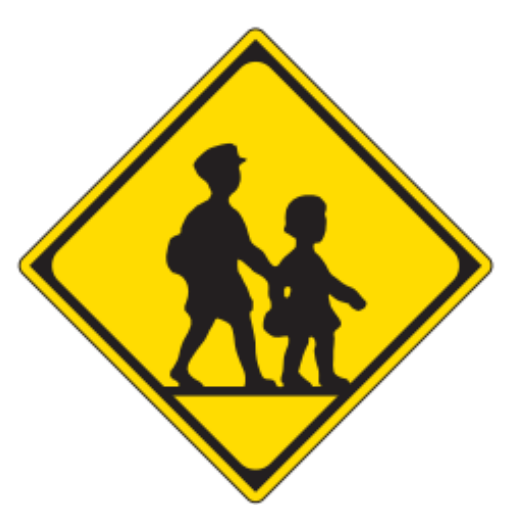

Fig. 9. School road sign

that the figure drawn in black was mixed with the QR code, making it difficult to detect.

In addition, the reasons why the QR code data can be read or not are considered as follows.

Camera focus and resolution: In most cases, traffic signs are installed on both sides of the road. If the focus near the center, the resolution will decrease on the shooting area's edges. Therefore, it becomes difficult to recognize the QR code unless the detection part follows the focus.

Vibration blur: The vibration blur occurs in experiment 1 when taking a video while walking. It is thought that the camera shake and vibration that also occur in the actual in-vehicle camera also affect the recognition of the QR code.

Reflection of light: When sunlight or car light is incident on the sign, the light is reflected on the polarizing film depending on the angle. In some instances, the QR code information will be missing, and it will be impossible to read. Furthermore, in the case of night, the changing of the sensor sensitivity and the shutter speed will cause the noise and the blur to increase. The image is distorted so that the $\mathrm{QR}$ code can be recognized, so the reading is considered impossible. 


\subsubsection{Experiment 2}

Considering the factors that make detection and reading difficult, we conducted Experiment 2. The high-resolution image taken by a $4 \mathrm{~K}$ camera was thought to contribute a high detection at a farther position. However, there is no significant difference even in the performance of the lens and sensor. It is considered that full HD video is suitable from the viewpoint of processing speed now.

\section{Conclusion}

In this study, the traffic sign detection system was proposed to not depend on external features by attaching a QR code using a polarizing film on the sign. The experimental result shows it was possible to detect and cut out the QR code position by the cascade classifier using Adaboost without any problem in most of the videos. However, only a few were able to read the QR code information in the cutout part. Regarding detection at night, noise and insufficient light intensity due to the sensor's high sensitivity made detection difficult. From the above, it is considered that recognizing road signs using a polarizing film is an effective method, but verification by a method other than QR code is required.

Although the amount of information that can be embedded may decrease, in future works, it is thought that recognition in a wide range of situations will be possible if a simplified pattern is used instead of the QR code.

\section{References}

(1) Evolution and dissemination of driving assistance technology and autonomous driving technology Ministry of Land, Infrastructure, Transport and Tourism, https:// www.mlit.go.jp/common/ $\$ 01213451 . p d f$, access date: 2020.11.10

(2) Overseas Trends (Part 2), Ministry of Land, Infrastructure, Transport and Tourism, https: //www.mlit.go.jp/road/ir/ir-council/ road_space/pdf03/03.pdf, access date: 2020.11.10

(3) Explanation of QR code detection API, Aki Teshima, https://qiita.com/tomoaki_teshima/items/ f136e51fa1da6927471b, access date: 2020.11 .12

(4) Y-Kashiwase(2011), "Enjoy experiments and explore the wonders of light Introductory optics[Translated from Japanese]", Bookcom Co., Ltd., pp.106-110

(5) R-Kurazume(2008), "-CVIM Tutorial Series-Computer Vision State-of-the-art Guide 1[Translated from Japanese]", Adcom Media Co., Ltd., pp.139-143
(6) Cascade Classifier Training, https: //docs.opencv.org/3.4/dc/d88/ tutorial_traincascade.html , access date: 2020.11.10

(7) Haar Feature-based Cascade Classifier for Object Detection, Opencv official documentation, https: //docs.opencv.org/2.4/modules/objdetect/doc/ cascade_classification.html\#cascadeclassifierdetectmultiscale, access date: 2020.11.10 